\title{
LeGes
}

Michael Strebel

\section{Die Praxis des Solothurner Verordnungsvetos}

Das Kantonsparlament von Solothurn ist die einzige Legislative mit einem umfassenden Veto bei Verordnungen und deren Änderungen. Es ist ein Instrument, das nur bei einer Minderheit der Verordnungen zum Tragen kommt und - so zeigt es die Praxis in Solothurn - nicht zu Blockaden oder relevanten Verzögerungen führt. Dies liegt auch an den gesetzlichen Rahmenbedingungen des Vetos. Durch die Möglichkeit des Vetos wird der parlamentarische Entscheidungskorridor vergrössert. Dieser Artikel zeigt die Praxis des Solothurner Vetos anhand von konkreten Beispielen auf.

Beitragsart: Wissenschaftliche Beiträge

Zitiervorschlag: Michael Strebel, Die Praxis des Solothurner Verordnungsvetos, in: LeGes 32 (2021) 2 


\section{Inhaltsübersicht}

1. Einleitung

2. Grundlagen des Solothurner Verordnungsvetos

3. Praxis des Solothurner Verordnungsvetos

3.1. Destruktiv oder konstruktiv?

3.2. Verordnungen und Verordnungsvetos

3.2.1. Verordnung ohne Einspruch

3.2.2. Einspruch gegen eine Verordnung abgelehnt

3.2.3. Einspruch gegen eine Verordnung, Bestätigung durch die Legislative und Rückzug der Verordnung

3.2.4. Einspruch gegen eine Verordnung und Bestätigung durch den Kantonsrat

a. Verabschiedung der Verordnung und Zustellung an die Legislative

b. Drei Einsprüche, eine Bestätigung

c. Wiederholung des Vorgangs

d. Ablehnung des Einspruchs und Inkraftsetzung

e. Würdigung

3.3. Das Verordnungsveto im Kontext der Bekämpfung der Covid-19-Pandemie

4. Fazit

\section{Einleitung}

[1] Das Verordnungsveto hat einen schwierigen Stand. Erst kürzlich lehnte die Bundesversammlung ein solches ab (2020), was sich in ablehnende Beschlüsse einiger Kantonsparlamente einreiht: Zürich (2017; der parlamentarische Vorstoss wurde allerdings zurückgezogen), Schaffhausen (2016), St. Gallen (2013), Basel-Stadt (2011), Aargau (2010) sowie Zug (2010).

[2] So verbleibt der Solothurner Kantonsrat weiterhin als (fast) einzige kantonale Legislative mit einem Verordnungsveto, sicherlich jedoch mit dem umfassendsten. Sein Verordnungsveto wird näher beleuchtet. Im Zentrum der Analyse steht dabei die Praxis und nicht eine grundsätzliche staatspolitische Auseinandersetzung zu Pro und Kontra eines Verordnungsvetos; zu dieser Frage gibt es bereits einige Publikationen.

[3] Zunächst wird auf die Einführung dieses parlamentarischen Instrumentes eingegangen. Danach wird der Blick auf die Verfassungs- und Gesetzesgrundlagen gerichtet. Anschliessend wird eine Auslegeordnung anhand konkreter Verordnungen vorgenommen. Zudem wird auf eine aktuelle Entwicklung in Bezug auf die Entwicklung im Zusammenhang mit der Bekämpfung der Covid-19-Pandemie mittels Notverordnungen hingewiesen. Der Artikel schliesst mit einem Fazit ab.

\section{Grundlagen des Solothurner Verordnungsvetos}

[4] Das «Solothurner Veto» erstreckt sich auf sämtliche Verordnungen der Regierung und Gerichte; dadurch unterscheidet es sich vom Vetorecht des Freiburger Kantonsparlamentes, das nicht für alle Verordnungen gilt: Delegiert das Freiburger Parlament in einem Erlass Gesetzgebungskompetenzen an die Exekutive und beabsichtigt es, deren Umsetzung zu kontrollieren, so entscheidet es jeweils von Fall zu Fall (Auer 2016, 301), ob es eine Vetoklausel einfügen will (Art. 93 Abs. 3 KV FR, SGF 10.1). Spätere Änderungen der Verordnungen sind jedoch nicht davon betroffen (AUER 2016, 301). 
[5] Im Kanton Wallis ist das Kantonsparlament ebenfalls befugt, in gesetzgeberischen Erlassen mit Delegation anzugeben, ob die Verordnung der Genehmigung des Kantonsparlaments unterliegen soll (Art. 57 Abs. 2 KV VS, SGS 101.1; Auer 2016, 300). Dort wurde dieses Recht auch tatsächlich schon des Öfteren genutzt; allerdings kam es noch nie vor, dass eine Verordnung nicht genehmigt wurde (Auer 2016, 301; Baumann 2010, 17).

[6] Doch zurück zum umfassenden Verordnungsveto des Solothurner Kantonsrates. Das Verordnungsveto wurde im Rahmen der Totalrevision der Kantonsverfassung (KV) mit Inkrafttreten im Jahr 1988 eingeführt.

[7] Der parlamentarische Ablauf stellt sich wie folgt dar: Der Regierungsrat erlässt Verordnungen auf der Grundlage und im Rahmen der Gesetze, Staatsverträge und Konkordate. 17 Kantonsräte (ein Sechstel des gesamten Parlaments) können innert 60 Tagen gegen eine vom Regierungsrat beschlossene Verordnung oder deren Änderung Einspruch einlegen. Der Einspruch ist kurz zu begründen. Bestätigt die Mehrheit der anwesenden Kantonsräte den Einspruch, so wird die Vorlage an den Regierungsrat zurückgewiesen - dabei hat das Veto eine rein kassatorische Wirkung. Die Verfassungs-, die Gesetzes- und die Reglementsbestimmungen zum Verordnungsveto sind in der Tabelle 1 aufgeführt.

Tabelle 1: Verfassungs-, Gesetzes- und Reglementsbestimmungen zum Solothurner Verordnungsveto

\begin{tabular}{|l|l|}
\hline Rechtsquelle & Bestimmung \\
\hline Kantonsverfassung & $\begin{array}{l}\text { Art. 79 Rechtsetzung } \\
\ldots\end{array}$ \\
& $\begin{array}{l}\text { 2 Der Regierungsrat erlässt Verordnungen auf der Grundlage und im } \\
\text { Rahmen der Gesetze, Staatsverträge und Konkordate. } \\
3 \text { 17 Kantonsräte können innert 60 Tagen gegen eine vom Regierungsrat } \\
\text { beschlossene Verordnung oder Verordnungsänderung Einspruch } \\
\text { einlegen. Wird der Einspruch durch die Mehrheit der anwesenden } \\
\text { Kantonsräte bestätigt, so ist die Vorlage an den Regierungsrat } \\
\text { zurückgewiesen. Das Kantonsratsgesetz regelt das nähere Verfahren. } \\
4 \text { Der Regierungsrat kann überdies Verordnungen erlassen, um } \\
\text { eingetretenen oder unmittelbar drohenden Störungen der öffentlichen } \\
\text { Ordnung und Sicherheit sowie sozialen Notständen zu begegnen. } \\
\text { Solche Verordnungen sind sofort durch den Kantonsrat genehmigen zu } \\
\text { lassen. Sie fallen spätestens ein Jahr nach Inkrafttreten dahin. }\end{array}$ \\
\hline
\end{tabular}




\begin{tabular}{|c|c|}
\hline Rechtsquelle & Bestimmung \\
\hline Kantonsratsgesetz & $\begin{array}{l}\S 44 \text { Verordnungsveto } \\
{ }^{1} \text { Hat der Regierungsrat eine Verordnung oder Verordnungsänderung } \\
\text { beschlossen, stellt er den Text den Ratsmitgliedern zu. } \\
{ }^{2} \text { Innert } 60 \text { Tagen seit Beschlussdatum kann jedes Ratsmitglied gegen } \\
\text { die Verordnung oder Verordnungsänderung schriftlich Einspruch } \\
\text { erheben. Findet in dieser Zeit keine Session statt, verlängert sich die } \\
\text { Einspruchsfrist bis zum letzten Tag der nächsten Session. Der } \\
\text { Einspruch ist kurz zu begründen. } \\
{ }^{3} \text { Erheben mindestens } 17 \text { Ratsmitglieder Einspruch, entscheidet der } \\
\text { Rat über die Bestätigung des Einspruchs in der Regel in der nächsten } \\
\text { Session. } \\
{ }^{4} \text { Die Absätze 1-3 gelten sinngemäss auch für Verordnungen und } \\
\text { Geschäftsreglemente der Gerichte. }\end{array}$ \\
\hline $\begin{array}{l}\text { Geschäftsregle- } \\
\text { ment des } \\
\text { Kantonsrates }\end{array}$ & $\begin{array}{l}\S 90 \text { Verordnungsveto } \\
{ }^{1} \text { Ratsmitglieder, welche den Einspruch gegen eine Verordnung bzw. } \\
\text { eine Verordnungsänderung unterstützen wollen, sollen ihre } \\
\text { Unterschrift wenn möglich auf demselben Schriftstück leisten. }\end{array}$ \\
\hline
\end{tabular}

\section{Praxis des Solothurner Verordnungsvetos}

\subsection{Destruktiv oder konstruktiv?}

[8] Nachdem die rechtlichen Grundlagen aufgezeigt wurden, stellt sich unweigerlich die Frage, wie oft ein Veto durch die Parlamentsmitglieder ergriffen und letztlich auch bestätigt wird. Zu Beginn müssen jedoch nochmals zwei gegensätzliche Positionen aufgegriffen werden, die bei der Beratung im Verfassungsrat über das Verordnungsveto geäussert wurden:

«Wir haben immer noch den Eindruck, dieses Veto sei in erster Linie ein negatives Instrument - wir sagten etwa auch schon, es sei destruktiv, (...) sicher ist es nicht ein Instrument, mit dem wir etwas Positives zur Konfliktregelung oder Problemlösung beitragen können. Viel eher birgt es die Gefahr in sich, zusätzliche Konflikte zu schaffen. Wenn der Kantonsrat mit diesem Instrument eine Verordnung der Regierung blockiert, so kann dies Lähmungserscheinungen zur Folge haben, die nicht nur die Verordnung selbst betreffen, sondern häufig sicher auch das Gesetz, auf das sich die Verordnung abstützt» (Protokoll Verfassungsrat 1983, 1315).

[9] Demgegenüber stand:

«Das Veto kann zu einem konstruktiven Element werden. Man muss nicht nur daran denken, dass nachher das Veto ergriffen wird, um eine Verordnung bachab zu schi- 
cken. Es hat auch eine prophylaktische Wirkung. Der Regierungsrat muss sich überlegen, ob er mit seiner Verordnung am Kantonsrat vorbeipolitisiert. So geht es auch dem Kantonsrat, der sich überlegen muss, ob er am Volk vorbeipolitisiert. Ich kann mir durchaus vorstellen, dass sich bei der Erarbeitung der Verordnung eine konstruktive Zusammenarbeit ergibt, dies gerade wegen der Veto-Möglichkeit» (Protokoll Verfassungsrat 1983, 767).

[10] Destruktiv oder konstruktiv? Wie sieht es nun in der Praxis aus? Die Tabelle 2 gibt einen umfassenden Überblick seit der Einführung des Instruments bis Ende 2020.

[11] Folgende Punkte können herausgehoben werden:

- Insgesamt beschloss die Regierung 1191 Verordnungen.

- Seit Einführung des Verordnungsvetos 1988 erhob der Kantonsrat gegen durchschnittlich jede 15. Verordnung Einspruch.

- Die Anzahl der Einsprüche je Jahr schwankte jedoch stark: In einigen Jahren wurde kein einziger Einspruch erhoben, die maximale Anzahl der Einsprüche in einem Jahr lag bei 9 .

- Es können mehrere Einsprüche gegen eine Verordnung bzw. Verordnungsänderung eingehen. Teilweise wurden Einsprüche wieder zurückgezogen (diese werden jedoch in der Tabelle nicht ausgewiesen).

- Etwa jeder fünfte Einspruch wurde durch den Kantonsrat letztlich bestätigt.

- Das Verordnungsveto wird zurückhaltend angewendet.

- Der Regierungsrat wies von sich aus acht Verordnungen zurück.

Tabelle 2: Übersicht über die Praxis des Verordnungsvetos im Solothurner Kantonsrat

\begin{tabular}{|c|c|c|c|c|c|}
\hline Jahr & $\begin{array}{c}\text { Total } \\
\text { VO }\end{array}$ & $\begin{array}{c}\text { Total } \\
\text { Vetos }\end{array}$ & $\begin{array}{c}\text { Vom KR } \\
\text { abgelehnt }\end{array}$ & Vom KR bestätigt & $\begin{array}{c}\text { VO vom RR } \\
\text { zurückgezogen }\end{array}$ \\
\hline 1988 & 51 & 1 & 0 & 1 & 0 \\
\hline 1989 & 44 & 2 & 1 & 1 & 0 \\
\hline 1990 & 61 & 2 & 2 & 0 & 0 \\
\hline 1991 & 61 & 0 & 0 & 0 & 0 \\
\hline 1992 & 45 & 5 & 2 & 2 & 0 \\
\hline 1993 & 54 & 4 & 3 & 0 & 2 \\
\hline 1994 & 54 & 4 & 3 & 0 & 1 \\
\hline 1995 & 54 & 9 & 5 & 0 & 0 \\
\hline 1996 & 55 & 5 & 4 & 2 & 0 \\
\hline 1997 & 61 & 4 & 1 & 0 & 0 \\
\hline 1998 & 47 & 3 & 3 & 2 & 0 \\
\hline 1999 & 34 & 5 & 2 & 1 & 0 \\
\hline 2000 & 46 & 3 & 2 & 0 & 1 \\
\hline 2001 & 26 & 2 & 2 & 1 & 0 \\
\hline 2002 & 43 & 1 & 0 & & 0 \\
\hline 2003 & 31 & 3 & 2 & & 0 \\
\hline
\end{tabular}




\begin{tabular}{|c|c|c|c|c|c|}
\hline Jahr & $\begin{array}{c}\text { Total } \\
\text { VO }\end{array}$ & $\begin{array}{c}\text { Total } \\
\text { Vetos }\end{array}$ & $\begin{array}{c}\text { Vom KR } \\
\text { abgelehnt }\end{array}$ & Vom KR bestätigt & $\begin{array}{c}\text { VO vom RR } \\
\text { zurückgezogen }\end{array}$ \\
\hline 2004 & 26 & 1 & 1 & 0 & 0 \\
\hline 2005 & 41 & 3 & 2 & 1 & 0 \\
\hline 2006 & 42 & 3 & 2 & 0 & 0 \\
\hline 2007 & 25 & 0 & 0 & 0 & 0 \\
\hline 2008 & 18 & 0 & 0 & 0 & 0 \\
\hline 2009 & 34 & 2 & 0 & 0 & 2 \\
\hline 2010 & 30 & 7 & 4 & 1 & 0 \\
\hline 2011 & 25 & 4 & 0 & 2 & 0 \\
\hline 2012 & 17 & 2 & 1 & 0 & 0 \\
\hline 2013 & 24 & 0 & 0 & 0 & 0 \\
\hline 2014 & 22 & 1 & 1 & 0 & 0 \\
\hline 2015 & 29 & 1 & 1 & 0 & 0 \\
\hline 2016 & 15 & 0 & 0 & 1 & 0 \\
\hline 2017 & 24 & 2 & 1 & 0 & 0 \\
\hline 2018 & 11 & 1 & 1 & 0 & $\mathbf{8}$ \\
\hline 2019 & 19 & 0 & 0 & $\mathbf{1 7}$ & 0 \\
\hline 2020 & 22 & 1 & 1 & & 0 \\
\hline Total & $\mathbf{1 1 9 1}$ & $\mathbf{8 1}$ & $\mathbf{4 7}$ & & \\
\hline
\end{tabular}

Quelle: Erstellung durch die Parlamentsdienste; geringfügige eigene Anpassungen.

[12] Um zu illustrieren, wie die parlamentarische Praxis bei Verordnungsvetos ist, wird im Folgenden der Blick auf drei konkrete Verordnungen gerichtet.

\subsection{Verordnungen und Verordnungsvetos}

[13] Im Folgenden werden vier verschiedene Fälle exemplarisch dargestellt, welche sich durch einen unterschiedlichen Verlauf des Vetos auszeichnen. Eine Verordnung, gegen die:

- kein Einspruch erhoben wurde (3.2.1);

- Einspruch erhoben, jedoch durch den Kantonsrat abgelehnt wurde (3.2.2);

- Einspruch erhoben und durch den Kantonsrat bestätigt wurde, worauf der Regierungsrat die Verordnung zurückzog (3.2.3);

- Einspruch erhoben und durch den Kantonsrat bestätigt wurde (3.2.4).

[14] Bewusst wird bei der Analyse der Verordnungen die Datumsangabe der verschiedenen Stationen des Prozesses ausgewiesen. Dies ist von Bedeutung für die Einschätzung der Zeitspanne zwischen der Verabschiedung der Verordnung durch den Regierungsrat und der Inkraftsetzung. 


\subsubsection{Verordnung ohne Einspruch}

[15] Der Regierungsrat beschloss am 22. Juni 2020 eine Änderung der Verordnung über die Sitzungspauschalen, Anpassung der Entschädigung für Expertinnen und Experten von Qualifikationsverfahren der beruflichen Grundbildung (RRB Nr. 2020/928). Die Exekutive wollte die Entschädigungsansätze für Prüfungsexpertinnen und -experten der Berufsbildung ab dem Jahr 2021 erhöhen und den umliegenden Kantonen angleichen. Die Erhöhung der Ansätze hat jährliche Mehrkosten von 300000 Franken zur Folge.

[16] Nach dem Beschluss des Regierungsrates wurde die Öffentlichkeit über die Anpassung der Entschädigung bzw. die Änderung der Verordnung mittels Medienmitteilung «Zeitgemässe Entschädigung für Experten von Lehrabschlussprüfungen» (22.06.2020) informiert. Dies zeigt, welche Wichtigkeit und Bedeutung die Regierung der Verordnung inhaltlich zumass.

[17] Dem Kantonsrat wurde die Verordnung zugestellt mit dem Ablauf der Einspruchsfrist am 9. September 2020. Die Frist wird stets so gesetzt, dass sie nach einer Session endet (in diesem Beispiel dauerte die Session vom 1. September 2020 bis 9. September 2020), damit die Ratsmitglieder während der Session die Möglichkeit haben, die notwendigen Unterschriften zu sammeln, um Einspruch zu erheben. Die Frist lief allerdings ungenutzt ab.

[18] Die Staatskanzlei stellte dies fest (14. September 2020), publizierte die Änderung der Verordnung im Amtsblatt (18. September 2020) und teilte das Datum der Inkraftsetzung der Verordnung über die Sitzungsgelder und die Sitzungspauschalen (BGS 126.511.31) mit (1. Januar 2021).

[19] Der geschilderte Ablauf - insbesondere, dass kein parlamentarischer Einspruch erhoben wurde - ist das übliche Prozedere (siehe Tabelle 2).

\subsubsection{Einspruch gegen eine Verordnung abgelehnt}

[20] Am 1. Januar 2018 ist das solothurnische Einführungsgesetz zum Vollzug der eidgenössischen Umweltschutzgesetzgebung (BGS 812.1) in Kraft getreten. Damit konnte der Regierungsrat die Biosicherheitsverordnung (BioSV) erlassen.

[21] In der BioSV sollen die Zuständigkeiten und Verantwortlichkeiten für den Vollzug der Einschliessungsverordnung (SR 814.912) und der Freisetzungsverordnung (SR 814.911) im Kanton Solothurn geregelt sowie die Pflichten und Kompetenzen der Vollzugsstellen definiert werden. Insbesondere soll eine kantonale Kontakt-, Informations- und Koordinationsstelle für den Vollzug in Sachen gebietsfremde Organismen bezeichnet werden. Diese kurzen Abrisse zeigen die Komplexität der in der Verordnung geregelten Materie.

[22] Der Regierungsrat hat an seiner Sitzung vom 9. Dezember 2019 die BioSV beschlossen (RRB Nr. 2019/1974). Diese so verabschiedete Verordnung wurde dem Kantonsrat durch die Parlamentsdienste zugestellt mit dem vermerkten Ablauf der Einspruchsfrist am 7. Februar 2020.

[23] Im Rahmen der Januar-Session 2020 des Kantonsrates reichten 22 Parlamentsmitglieder ihren Einspruch gegen die BioSV ein, unter anderem mit der Begründung (die ausführlich dargelegt wurde), die Verordnung beinhalte zu viele Ausführungsunklarheiten (VET 0028/2020). Die Frist des Einspruchs wurde damit gewahrt und die Mindestanzahl, das Quorum von 17 Parlamentsmitgliedern, wurde erreicht. 
[24] Der so erfolgte Einspruch wurde anschliessend durch die Parlamentsdienste festgehalten und schriftlich der Regierung übermittelt mit der Einladung, rechtzeitig vor der nächsten MaiSession (5./6. Mai) schriftlich Stellung zu nehmen.

[25] Die Stellungnahme des Regierungsrates vom 25. Februar 2020 (RRB Nr. 2020/270) wurde den Parlamentsmitgliedern zugestellt und der Einspruch wurde für die Session traktandiert. Der Antrag der Exekutive, in diesem Fall auf Ablehnung des Einspruchs, wird generell nicht durch eine parlamentarische Kommission vorberaten. Die Stellungnahme geht folglich direkt ins Plenum zur abschliessenden Entscheidung.

[26] Der Kantonsrat lehnte in der Mai-Session nach eingehender Diskussion den Einspruch mit 49 zu 40 Stimmen bei 2 Enthaltungen ab. Der Regierungsrat setzte die BioSV (BGS 812.2) rückwirkend auf den 1. März 2020 in Kraft.

\subsubsection{Einspruch gegen eine Verordnung, Bestätigung durch die Legislative und Rückzug der Verordnung}

[27] Mit Beschluss vom 14. Dezember 2010 verabschiedete die Regierung die Übergangsverordnung zum Verfahren bei medizinischer Staatshaftung und setzte diese Verordnung per 1. Januar 2011 in Kraft. Damit wartete sie den Ablauf der Vetofrist von 60 Tagen nicht ab (dies ist ein eher seltener Vorgang).

[28] Es würde den Rahmen dieses Artikels sprengen, inhaltlich detailliert auf die Verordnung einzugehen. Für das Verständnis der eigentlichen Problematik ist dies auch nicht notwendig. Es genügt, die grundsätzliche Absicht zu erwähnen: Mit der Verordnung sollte anstelle des bisherigen verwaltungsrechtlichen Klageverfahrens im Bereich medizinischer Staatshaftung ein zweitinstanzliches Beschwerdeverfahren durch den Regierungsrat eingeführt werden. Gemäss Bundesgericht sollte im Bereich der Spitalhaftung ab 1. Januar 2011 ein doppelter Instanzenzug vorgesehen werden. Aus diesem Grund setzte die Regierung die Verordnung in Kraft, ohne das Ende der parlamentarischen Einspruchsfrist abzuwarten.

[29] Innerhalb der bis zum 18. Februar 2011 laufenden Einspruchsfrist wurden zwei Einsprüche erhoben. Es waren grundsätzliche Vorbehalte:

1. Die Regierung versucht, eine Verordnung durchzubringen, ohne sich auf ein geltendes Gesetz abstützen zu können (von 18 Parlamentsmitgliedern unterschrieben).

2. Die Verordnung widerspricht in allen Punkten Verfassung und Gesetz, ist damit rechtlich unzulässig und somit vollständig aufzuheben (von 38 Parlamentsmitgliedern unterschrieben).

[30] Die Exekutive wurde von den Parlamentsdiensten eingeladen, bis zum 1. März 2011 Stellung zu nehmen. Der Regierungsrat bat um Fristerstreckung, die gewährt wurde, und kam an seiner Sitzung vom 29. März 2011 auf seinen Beschluss vom 14. Dezember 2010 zurück. Er hat:

- die Verordnung zurückgezogen und

- eine neue Übergangsverordnung beschlossen (mit einer anderen Übergangslösung als in der ersten Verordnung, damit sollte Rechtssicherheit bestehen bis zum Vorliegen der definitiven Regelung auf Gesetzesstufe). 
[31] Die Frist für einen Einspruch gegen die Übergangsverordnung lief am 16. Juni 2011 ungenutzt ab. Die Übergangsverordnung zum Verfahren bei medizinischer Staatshaftung (BGS 124.22) trat rückwirkend auf den 1. Januar 2011 in Kraft, befristet bis zum Inkrafttreten der gesetzlichen Regelung (aufgehoben wurde die Verordnung per 22. Februar 2019).

\subsubsection{Einspruch gegen eine Verordnung und Bestätigung durch den Kantonsrat} a. Verabschiedung der Verordnung und Zustellung an die Legislative

[32] Der Regierungsrat beschloss am 2. Mai 2017 die Totalrevision der Jagdverordnung (RRB Nr. 2017/779), mit der parlamentarischen Einspruchsfrist bis zum 3. Juli 2017. Vorausgegangen war der Beschluss des Parlamentes im November 2016 zum neuen Jagdgesetz (BGS 626.11).

[33] Der Regierungsrat informierte die Öffentlichkeit mittels Medienmitteilung über das Ergebnis der Totalrevision: «Zeitgemässe Solothurner Jagdgesetzgebung unter Dach und Fach» (02.05.2017). Auf einen Punkt soll hingewiesen werden, weil dies die Legislative, die Exekutive sowie die Öffentlichkeit noch beschäftigte:

«(...) die Leinenpflicht für Hunde im Wald und in einem 100 Meter breiten Streifen ausserhalb des Waldes wird verlängert und soll neu vom 1. April bis zum 31. Juli gelten. Die bereits jetzt geltende Leinenpflicht im Mai und Juni wird dem geforderten Schutz der Wildtiere nicht gerecht, weil die Setz- und Brutzeit vieler einheimischer Wildtiere über diese Zeit hinaus dauert. Die neue Dauer der Leinenpflicht entspricht der Leinenpflichtdauer der Nachbarkantone Aargau, Basellandschaft und Luzern und soll verhindern, dass Hundehalterinnen oder Hundehalter dieser Nachbarkantone auf das Kantonsgebiet des Kantons Solothurn ausweichen, um ihre Hunde hier frei laufen zu lassen» (Medienmitteilung, 2.5.2017).

b. Drei Einsprüche, eine Bestätigung

[34] Gegen die Verordnung wurden im Rahmen der Juni-Session drei Einsprüche mit Datum 28. Juni 2017 erhoben (VET 0117a/2017, VET 0117b/2017, VET 0117c/2017). Die drei Einsprüche richteten sich gegen unterschiedliche Bestimmungen in der Verordnung.

[35] Der Regierungsrat nahm am 22. August 2017 zu jedem einzelnen Einspruch Stellung (RRB Nr. 2017/1412-1414). Er empfahl dem Parlament, alle drei abzulehnen. Die Stellungnahmen erfolgten wie vorgesehen rechtzeitig vor der September-Session der Legislative.

[36] Nun war das Parlament gefordert, über die Einsprüche zu entscheiden. Doch bevor es zur Abstimmung kam, zogen zwei Erstunterzeichner ihren Einspruch zurück. Somit verblieb ein Einspruch (VET 0117b/2017), bei dem die Legislative zu entscheiden hatte.

[37] Es lohnt sich, den Einspruch materiell zu betrachten, weil er den Punkt betrifft, welcher in der obigen Medienmitteilung erwähnt wurde, und weil er zu weiteren Diskussionen Anlass bot. Der Einspruch richtete sich konkret gegen $\S 4$ Abs. 1 der Hundeverordnung:

${ }^{1}$ Generelle Leinenpflicht herrscht

a) für alle Hunde

1. (geändert) im Wald und in einem 100 Meter breiten Streifen ausserhalb des Waldes vom 1. April bis 31. Juli; 
[38] Gegen diese Änderung der Hundeverordnung erhoben 18 Parlamentsmitglieder Einspruch mit folgender Begründung:

«Die bis jetzt geltenden Einschränkungen von 2 Monaten (Mai-Juni) ohne Angabe des erforderlichen Waldabstandes für die Hunde-Leinenpflicht sind schon heute fast nicht zu kontrollieren. Eine Ausdehnung dieser Pflicht ist unverhältnismässig und schlicht nicht nachvollziehbar, im Besonderen der geforderte Waldabstand von $100 \mathrm{~m}$ ist im Vollzug völlig unrealistisch» (VET 0117b/2017 1).

[39] Schliesslich stimmten 51 Parlamentsmitglieder für den Einspruch, 34 dagegen, 12 enthielten sich (3 waren abwesend). Somit wurde der Einspruch in der Parlamentssitzung vom 5. September 2017 bestätigt. Der Kantonsratsbeschluss (nicht referendumsfähig) wurde der Regierung übermittelt. Diese war nun (heraus)gefordert zu beurteilen, ob und wie sie mit dem Entscheid der Legislative umzugehen gedenkt.

\section{c. Wiederholung des Vorgangs}

[40] Mit Beschluss vom 26. September 2017 verabschiedete die Exekutive die Jagverordnung erneut (RRB Nr. 2017/1672). Sie ging auf den parlamentarischen Einspruch wie folgt ein:

[41] «Aufgrund dieses bestätigten Verordnungsvetos wird (...) auf die räumliche Ausdehnung der Leinenpflicht verzichtet, indem der Passus «... und in einem 100 Meter breiten Streifen ausserhalb des Waldes...) gestrichen wird. An der zeitlichen Ausdehnung der Leinenpflicht vom 1. April bis 31. Juli wird hingegen festgehalten. Diese Bestimmung ist aus biologischer Sicht sehr sinnvoll. Ausserdem erfolgt dadurch eine Harmonisierung der Dauer der Leinenpflicht mit den Nachbarkantonen Aargau und Basel-Landschaft. Dem Ausführen von Hunden aus Nachbarkantonen («Hundetourismus») wird dadurch entgegengewirkt» (RRB Nr. 2017/1672, 1). Über den regierungsrätlichen Beschluss wurde mittels Medienmitteilung «Jagdverordnung ohne Leinenpflicht ausserhalb des Waldes» (26.09.2017) informiert.

[42] Die Einspruchsfrist endete am 27. November 2017. Erneut wurde in der November-Session Einspruch erhoben, diesmal von 30 Parlamentsmitgliedern. Der Einspruch enthielt vier Punkte:

1. «Die fragliche Änderung war bereits Gegenstand des Vetos (...). Dieses Veto wurde vom Kantonsrat (...) deutlich gutgeheissen. Das Veto ist ein Einspruchsrecht mit kassatorischer Wirkung. Mit der Gutheissung des Vetos ist die beanstandete Norm kassiert (aufgehoben). Dass der Regierungsrat sich über dieses kantonsrätliche Veto hinwegsetzt und an der kassierten Bestimmung in leicht abgeschwächter Form festhält, ist eine klare Missachtung der Kompetenznorm in $\S 44$ des Kantonsratsgesetzes.

2. Die beanstandete Norm verletzt den Grundsatz, dass eine Gesetzes- und damit auch eine Verordnungsänderung notwendig und verhältnismässig sein muss. Die Verdoppelung der Leinenpflicht ist weder das eine noch das andere. Sie schränkt die Bewegungsfreiheit des Menschen, welcher sich mit einem Hund in der Gegend bewegt, grundlos weiter ein. Sie verstösst zudem gegen den Grundsatz (...) der eidgenössischen Tierschutzverordnung. (...).

3. Für jagende oder wildernde Hunde besteht bereits jetzt eine absolute und ständige Leinenpflicht $(\ldots)$. 
4. Es geht wohlgemerkt vorliegend nur um die Verdoppelung der absoluten Leinenpflicht im Wald. Aber ganz nebenbei bemerkt, gehört dieses Thema nicht in die Hundeverordnung. Diese regelt die Hundehaltung. Der Schutz von Wildtieren in diesem Zusammenhang gehört in das Jagdgesetz» (VET 0198/2017, 1).

\section{d. Ablehnung des Einspruchs und Inkraftsetzung}

[43] Die Regierung nahm vor der anstehenden Dezember-Session Stellung erneut zum Einspruch: «In der Begründung zum Veto (...) wird vor allem der geforderte Waldabstand von $100 \mathrm{~m}$ beanstandet. Ob sich das Veto nur gegen den vorgesehenen Waldabstand oder auch gegen die zeitliche Ausdehnung der Leinenpflicht richtet, geht weder aus dem Wortlaut noch aus der kantonsrätlichen Debatte klar hervor. Davon ausgehend, dass sich das Veto (...) nur gegen den vorgesehenen Waldabstand richtet, haben wir in der Neufassung der betreffenden Verordnungsbestimmung auf die Vorgabe eines Waldabstandes verzichtet. Durch die vorgesehene Neuregelung der Leinenpflicht wird weder in die Gesetzgebungskompetenz des Kantonsrats eingegriffen noch dessen gesetzgeberischer Wille untergraben» (RRB Nr. 2017/2003, 2). Die Regierung lehnte folglich den Einspruch ab.

[44] Nach einer intensiv geführten Parlamentsdebatte stimmten 30 Parlamentsmitglieder für die Unterstützung des Vetos, 65 dagegen. Nach diesem Entscheid konnte die Regierung die Jagdverordnung (BGS 626.12) per 1. Januar 2018 in Kraft setzen.

\section{e. Würdigung}

[45] Der geschilderte Vorgang («Pingpong» zwischen Legislative und Exekutive) beim Veto zur Jagdverordnung ist unüblich. Dennoch wurde gerade dieser Vorgang als negatives Beispiel herangezogen, um zu begründen, warum ein legislatives Veto - auch auf Bundesebene - abzulehnen sei. So schrieb die Solothurner Zeitung zu der Zeit, als der Nationalrat über das Vetorecht debattierte, mit der Überschrift «Die Regierung an der kurzen Leine: Muss die Machtfülle des Bundesrats beschränkt werden, wenn er sich um den Willen des Gesetzgebers foutiert? Als einziger Kanton kennt Solothurn ein Vetorecht. Die Erfahrungen sind zwiespältig.» Um dann die Jagdverordnung heranzuziehen: «Das Solothurner Hickhack um die Leinenpflicht offenbart ein Problem, über das der Nationalrat ebenfalls debattiert hat: Führt das Verordnungsveto zu Unsicherheiten?» (01.07.2019).

[46] Über alle 1191 Verordnungen hinweg kann diesbezüglich Entwarnung gegeben werden.

[47] Es war in der Praxis des Solothurner Vetos ein singulärer Fall, und selten fand eine Verordnung eine so grosse mediale Beachtung wie die Jagdverordnung. Es liegt sodann in der (politischen) Beurteilung, ob aus dem Einzelfall generelle Schlüsse gezogen werden (sollen).

[48] Jedoch typisch an der Jagdverordnung war, dass sehr schnell nicht mehr der inhaltliche Einspruch im Vordergrund stand, sondern die Frage, ob die Legislative überhaupt dazu berechtigt sei. Für die Regierung war eindeutig: Das vorliegende Veto zur Jagdverordnung schiesst

«über den vorgesehenen Inhalt des Verordnungsvetos hinaus. Lehnt das Veto eine Verordnungsänderung ab, nur weil es einen Sachverhalt anders geregelt haben will, wird es nicht nur als Einspruch gegen einen Erlass oder gegen eine Änderung einer Ver- 
ordnung eingesetzt, sondern als Gestaltungsmittel der Legislative, ohne dass eine Änderungsabsicht der Exekutive vorliegt. Sollte dieser weiter gefasste Anwendungsbereich des Verordnungsvetos durch den Kantonsrat bestätigt werden, sehen wir darin einen Übergriff der Legislative in den Wirkungsbereich der Exekutive, was als Verletzung der Gewaltenteilung und damit als Verfassungsverletzung gewertet werden muss» (RRB 2017/1413, 2).

[49] Dieser regierungsrätlichen Position ist entgegenzuhalten: Erstens ist das legislative Vetorecht an keine inhaltlichen Bedingungen gebunden (BRECHвüHL 2010). Weder auf Verfassungsstufe noch auf Gesetzesstufe gibt es entsprechende Vorgaben. Man kann den Einspruch als (politisch) falsch, zu kleinteilig, zu detailliert beurteilen; einen legislativen Verfassungsbruch stellt er aber nicht dar.

[50] Die Regierung konnte die Jagdverordnung, sieben Monate nachdem sie der Legislative erstmals zugestellt worden war, in Kraft setzen. Wichtig ist der Hinweis, dass diese Verordnung, wenn es zu keinem Verordnungsveto gekommen wäre, auf denselben Zeitpunkt in Kraft gesetzt worden wäre. Auf der Zeitachse hat sich durch die (mehrmaligen) Einsprüche nichts verändert; es gab folglich keine zeitliche Verzögerung.

\subsection{Das Verordnungsveto im Kontext der Bekämpfung der Covid-19- Pandemie}

[51] Aus aktuellem Anlass wird das Verordnungsveto im Kontext der Bekämpfung der Covid-19Pandemie betrachtet.

[52] Gemäss der Solothuner Verfassung kann die Regierung Verordnungen erlassen, um eingetretenen oder unmittelbar drohenden Störungen der öffentlichen Ordnung und Sicherheit sowie sozialen Notständen zu begegnen. Solche Verordnungen sind sofort durch den Kantonsrat genehmigen zu lassen. Sie fallen spätestens ein Jahr nach Inkrafttreten dahin (Art. 79 Abs. 4 KV, BGS 111.1).

[53] Da die Regierung in verschiedenen Politikfeldern unverzüglichen Handlungsbedarf ausmachte, erliess sie die entsprechenden Notverordnungen (gemäss Art. 79 Abs. 4 KV). Aus Sicht der Exekutive musste sie diesen Weg beschreiten, weil «normale» Verordnungen unter dem üblichen Vorbehalt des Einspruches stehen und erst nach Ablauf der unbenutzten Einspruchsfrist bzw., wenn Einspruch erhoben würde, nach dem Entscheid der Legislative in Kraft gesetzt werden können. Lediglich zwei Verordnungen wurden nicht als Notverordnungen erlassen, nämlich die Verordnung über Massnahmen im Kulturbereich zur Bewältigung der Covid-19-Pandemie (Einspruchsfrist lief ungenutzt ab (BGS 431.118)) sowie die Verordnung über die Festlegung des Zinssatzes für die Verzugszinsen bei den Haupt- und Nebensteuern infolge der COVID-19Pandemie (Einspruchsfrist lief ungenutzt ab, Verordnung zwischenzeitlich wieder aufgehoben (BGS 105.1)).

[54] Die Tabelle 3 zeigt alle Notverordnungen, die zur Bekämpfung von Covid-19 erlassen wurden. Allerdings wurde lediglich die erste Verordnung auch als Notverordnung bezeichnet. Bei den nachfolgenden Verordnungen hat die Regierung darauf verzichtet, diese so zu benennen, auch wenn es sich faktisch um Notverordnungen handelte. Es waren in der Anzahl einige Notverordnungen, die teilweise in kurzer Frist einmal oder mehrfach geändert wurden. Für die Legislative bestand die enorme Herausforderung, die Notverordnungen inkl. der Änderungen einerseits un- 
verzüglich in den zuständigen parlamentarischen Kommissionen (unter erschwerten Coronabedingungen) vorzubereiten, damit das Plenum auch sofort, wie in der Kantonsverfassung vorgegeben, darüber entscheiden konnte. Wie in der Tabelle ersichtlich, verging dennoch teilweise mehr als ein Monat, bis die Verordnung durch die Legislative genehmigt wurde. Diese längere Zeitspanne hatte jedoch keinen Einfluss auf den Zeitpunkt der Inkraftsetzung der Notverordnung: Die Exekutive hat die Verordnungen i. d. R. unmittelbar nach dem Regierungsbeschluss in Kraft gesetzt. Die Situation trat nicht ein, dass die Legislative eine bereits in Kraft gesetzte Notverordnung nachträglich ablehnte.

[55] Der Weg über Notverordnungen hatte bestimmt einen positiven Effekt: Die parlamentarische Debatte darüber war demokratiepolitisch bedeutend, auch wenn der Kantonsrat inhaltlich nichts mehr an den Notverordnungen ändern konnte. Entscheide des Parlaments werden in der Öffentlichkeit gehört, sie erzeugen dadurch eine hohe Legitimität - das ist gerade in schwierigen Situationen eine Qualität der parlamentarischen Demokratie (Strebel 2021).

[56] In der vorgelagerten Beratung in den zuständigen (bestimmte Verordnungen in mehreren) parlamentarischen Kommissionen konnten die Verordnungen mit den zuständigen Mitgliedern der Regierung diskutiert werden.

[57] Diese Ausführungen über das (zeitlich lange) Regieren über Notverordnungen im Kontext des Verordnungsvetos sind nicht abschliessend; es braucht mit etwas zeitlichem Abstand weitere Auswertungen und Analysen.

Tabelle 3: Überblick der Notverordnungen

\begin{tabular}{|c|c|c|}
\hline $\begin{array}{r}\text { Beschluss RR } \\
\text { - Änderung }\end{array}$ & Notverordnung & Beschluss KR \\
\hline $\begin{array}{r}23.03 .2020 \\
\text { - } 01.04 .2020\end{array}$ & $\begin{array}{l}\text { Notverordnung über die Überbrückungshilfe } \\
\text { für Selbstständigerwerbende infolge der } \\
\text { Corona-Pandemie (Beschluss-Nr. 2020/432, } \\
\text { 2020/525) }\end{array}$ & 05.05 .2020 \\
\hline 24.03 .2020 & $\begin{array}{l}\text { Verordnung zur Sicherstellung der } \\
\text { Handlungsfähigkeit der Gemeinden aufgrund } \\
\text { der Massnahmen zur Bekämpfung des } \\
\text { Coronavirus (COVID-19) (CorGeV) } \\
\text { (Beschluss-Nr. 2020/475) }\end{array}$ & 05.05 .2020 \\
\hline 24.03 .2020 & $\begin{array}{l}\text { Verordnung über den Fristenstillstand bei } \\
\text { kantonalen Volksbegehren aufgrund der } \\
\text { Massnahmen zur Bekämpfung des Coronavirus } \\
\text { (COVID-19) (Beschluss-Nr. 2020/487) }\end{array}$ & 05.05 .2020 \\
\hline 07.04 .2020 & $\begin{array}{l}\text { Verordnung zur Sicherung von } \\
\text { Ergänzungsleistungen für } \\
\text { einkommensschwache Familien während der } \\
\text { Corona-Pandemie (Beschluss-Nr. 2020/526) }\end{array}$ & 05.05 .2020 \\
\hline
\end{tabular}




\begin{tabular}{|c|c|c|}
\hline $\begin{array}{l}\text { Beschluss RR } \\
\text { • Änderung }\end{array}$ & Notverordnung & Beschluss KR \\
\hline 16.04 .2020 & $\begin{array}{l}\text { Verordnung über die Abfederung der } \\
\text { wirtschaftlichen Auswirkungen des } \\
\text { Coronavirus (COVID-19) im Kultursektor } \\
\text { (CorKulturV) (Beschluss-Nr. 2020/529) }\end{array}$ & 05.05 .2020 \\
\hline 21.04 .2020 & $\begin{array}{l}\text { Verordnung zur Abfederung der } \\
\text { wirtschaftlichen Folgen der Massnahmen zur } \\
\text { Bekämpfung des Coronavirus (COVID-19) bei } \\
\text { Kindertagesstätten (Beschluss-Nr. 2020/599) }\end{array}$ & 05.05 .2020 \\
\hline 19.05 .2020 & $\begin{array}{l}\text { Verordnung über die Ergänzungsprüfung } \\
\text { Passerelle Berufsmaturität oder Fachmaturität - } \\
\text { universitäre Hochschulen im Schuljahr } \\
\text { 2019/2020 aufgrund des Coronavirus } \\
\text { (COVID-19) (CorPasserelleV) (Beschluss-Nr. } \\
\text { 2020/769) }\end{array}$ & 01.07 .2020 \\
\hline 19.05 .2020 & $\begin{array}{l}\text { Verordnung über die Abschlussprüfungen an } \\
\text { den Fachmittelschulen im Schuljahr 2019/2020 } \\
\text { aufgrund des Coronavirus (COVID-19) } \\
\text { (CorFMS-V) (Beschluss-Nr. 2020/770) }\end{array}$ & 01.07 .2020 \\
\hline 26.05 .2020 & $\begin{array}{l}\text { Verordnung über die Finanzierung von } \\
\text { zusätzlichen Schülertransporten aufgrund des } \\
\text { Coronavirus (COVID-19) (CorST-V) } \\
\text { (Beschluss-Nr. 2020/794) }\end{array}$ & 01.07 .2020 \\
\hline 30.06 .2020 & $\begin{array}{l}\text { Verordnung zur Abfederung der } \\
\text { wirtschaftlichen Folgen der Massnahmen zur } \\
\text { Bekämpfung des Coronavirus (COVID-19) bei } \\
\text { Miet- und Pachtzinsen für Geschäftsräume } \\
\text { (Beschluss-Nr. 2020/1023) }\end{array}$ & 01.09 .2020 \\
\hline $\begin{array}{l}21.10 .2020 \\
\qquad \begin{aligned} \text { - } 26.10 .2020 \\
\text { - } 30.10 .2020 \\
\text { - } 08.12 .2020 \\
\text { - } 22.12 .2020 \\
\text { - } 14.01 .2021 \\
\text { - } 16.04 .2021\end{aligned}\end{array}$ & $\begin{array}{l}\text { Verordnung über Massnahmen des Kantons } \\
\text { Solothurn zur Bekämpfung der } \\
\text { Covid-19-Epidemie (V Covid-19) (Beschluss-Nr. } \\
\text { 2020/1474, 2020/1476, 2020/1510, 2020/1785, } \\
\text { 2020/1890, 2021/34, 2021/513). }\end{array}$ & $\begin{array}{l}11.11 .2020 \\
15.12 .2020 \\
27.01 .2021 \\
12.05 .2021\end{array}$ \\
\hline 30.10 .2020 & $\begin{array}{l}\text { Verordnung } 2 \text { zur Sicherstellung der } \\
\text { Handlungsfähigkeit der Gemeinden aufgrund } \\
\text { der Massnahmen zur Bekämpfung des } \\
\text { Coronavirus (CorGeV 2) (Beschluss-Nr. } \\
\text { 2020/1509) }\end{array}$ & 11.11 .2020 \\
\hline
\end{tabular}




\begin{tabular}{|c|c|c|}
\hline $\begin{array}{l}\text { Beschluss RR } \\
\text { • Änderung }\end{array}$ & Notverordnung & Beschluss KR \\
\hline $\begin{array}{l}24.11 .2020 \\
\text { • } 27.04 .2021\end{array}$ & $\begin{array}{l}\text { Verordnung über die Unterstellung von } \\
\text { Schutzeinrichtungen vor Gastronomiebetrieben } \\
\text { unter } \S 4 \text { der Kantonalen Bauverordnung (KBV; } \\
\text { BGS 711.61) infolge der Corona-Pandemie } \\
(\text { CorSE-V) (Beschluss-Nr. 2020/1665, 2021/579) }\end{array}$ & $\begin{array}{l}15.12 .2020 \\
12.05 .2021\end{array}$ \\
\hline $\begin{array}{l}07.12 .2020 \\
\begin{aligned} \text { - } 15.12 .2020 \\
\text { - } 24.12 .2020 \\
\text { - } 19.01 .2021 \\
\text { - } 15.02 .2021 \\
\text { - } 11.03 .2021 \\
\text { - } 27.04 .2021 \\
\text { - } 25.05 .2021\end{aligned}\end{array}$ & $\begin{array}{l}\text { Verordnung über Härtefallmassnahmen für } \\
\text { Unternehmen im Zusammenhang mit Covid-19 } \\
\text { (Härtefallverordnung-SO) (Beschluss-Nr. } \\
\text { 2020/1784, 2020/1843, 2020/1899, 2021/69, } \\
2021 / 150,2021 / 308,2021 / 602,2021 / 728 \text { ) }\end{array}$ & $\begin{array}{l}16.12 .2020 \\
27.01 .2021 \\
03.03 .2021 \\
12.05 .2021 \\
07.07 .2021\end{array}$ \\
\hline
\end{tabular}

Bemerkungen: Eigene Auswertung (Stand der Auswertung: 7.7.2021); teilweise sind die Verordnungen wieder aufgehoben, spätestens jedoch ein Jahr nach dem Inkrafttreten (die Aufhebung wurde nicht vermerkt). Verordnungen wurden auch verlängert; dazu brauchte es einen Regierungs-, aber keinen Parlamentsbeschluss (die Verlängerung ist in der Tabelle nicht ausgewiesen). Die Verordnungen und die dazugehörigen RRB können hier abgerufen werden: https://rrb.so.ch; die Parlamentsbeschlüsse hier: https://so.ch/ parlament/geschaefte/extranet-archiv.

\section{4. $\quad$ Fazit}

[58] Das Solothurner Verordnungsveto wurde in so manchen Legislativen als Vorbild genommen, um einen entsprechenden parlamentarischen Vorstoss einzureichen. Jedoch nüchtern betrachtet: Ein Exportartikel, der auch Abnehmer findet, ist das Veto nicht.

[59] An der Jahrestagung der Schweizerischen Gesellschaft für Parlamentsfragen mit dem Schwerpunktthema Verordnungsveto ${ }^{1}$ zog der Solothurner Kantonsrat Peter Hodel ${ }^{2}$ in seinem Referat über das «Solothurner Veto» aufgrund seiner langjährigen parlamentarischen Erfahrung folgende Bilanz:

- Von Blockaden kann keine Rede sein.

- Das Solothurner Veto erweitert zwar in bescheidenem Umfang den Einflussbereich des Parlamentes. Dieses kann aber mit dem Veto nur eine Zurückweisung erwirken, aber keine inhaltliche Abänderung direkt vornehmen.

1 Alle Referate und die anschliessende Podiumsdiskussion können hier nachgelesen werden: parlament-2019-3.

2 Peter Hodel wurde am 23.4.2021 in die Solothurner Regierung gewählt. 
- Andererseits hat unser Veto noch den guten Nebeneffekt, dass damit das Bedürfnis bei der Beratung der Gesetzesvorlage, auf Stufe Legislative bereits die Struktur oder den Inhalt der Verordnung zu kennen, deutlich kleiner ist. (Hodel 2019, 4).

[60] Welche Anhaltspunkte kann die Solothurner Praxis bieten, wenn eine Legislative ein Veto in seine Gesetzgebungslandschaft einführen will?

[61] Die Kompetenzverteilung zwischen Exekutive und Legislative sollte auf verfassungsrechtlichen Grundlagen fussen bzw. in der Kantonsverfassung festgeschrieben sein.

[62] Zudem sollten zwei Hürden eingebaut werden: Zum einen ist die Anzahl der Unterschriften für die Einreichung des Einspruchs gegen die Verordnung festzulegen. Zum anderen muss die Mehrheit des Parlamentes den Einspruch bestätigen.

[63] Obendrein gilt es den parlamentarischen Einspruch zu begründen.

[64] Durch die Möglichkeit eines solchen Vetos wird der parlamentarische Entscheidungskorridor vergrössert (Strebel 2017). Dies wiederum würde zu einer partiellen Stärkung des Parlamentes führen, ohne jedoch die Exekutive in ihren Handlungs- und Entscheidungskompetenzen zu beschränken oder den Prozess der Inkraftsetzung von Verordnungen - wenn überhaupt - signifikant zu verzögern.

Michael Strebel, promovierter Politikwissenschaftler, war Ratssekretär des Solothurner Kantonsrates und beschäftigt sich wissenschaftlich und beruflich mit Parlamenten. U. a. hat er Lehraufträge an der Fernuniversität Hagen und an der Universität Freiburg im Breisgau.

\section{Literaturverzeichnis}

Auer, Andreas (2016): Staatsrecht der schweizerischen Kantone, Stämpfli Verlag, Bern.

Baumann, Claude (2010): Die Genehmigungsverordnung im Kanton Wallis, in: Parlament, 2/2010, S. 17-18.

BrechiüHL, Fritz (2010): Verordnungsveto des Solothurner Kantonsrates, in: Parlament, 2/2010, S. 8-12.

Hodel, Peter (2019): Sollen Parlamente auf die Verordnungsgebung der Regierung Einfluss nehmen? Erfahrungen aus dem Kanton Solothurn, in: Parlament, 3/2019, S. 3-4.

Solothurner Zeitung:

- Verordnungsveto spaltet die Standesstimme, 28.09.2019.

- Die Regierung an der kurzen Leine, 01.07.2019, S. 5.

- Hunde müssen wieder länger an die Leine, 27.09.2017, S. 1.

- Übergangen [Kommentar], 27.09.2017, S. 1.

- Wer sündigt denn hier?, 27.09.2017, S. 21.

- Das Verordnungsveto: Einzig- und sehr eigenartig, 27.09.2017, S. 21.

Strebel, Michael (2017): Beitrag zur aktuellen Debatte zum parlamentarischen Verordnungsveto, in: Parlament, 2/2017, S. $31-42$.

Strebel, Michael (2021): «Parlament funktioniert auch in der Krise», in: Solothurner Zeitung: 06.01.2021, S. 15. 